\title{
Role of circRNAs in viral infection and their significance for diagnosis and treatment (Review)
}

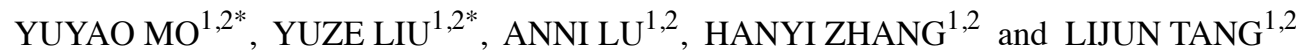 \\ ${ }^{1}$ School of Life Science and ${ }^{2}$ Xiangya School of Medicine, Central South University, Changsha, Hunan 410013, P.R. China
}

Received December 22, 2020; Accepted March 8, 2021

DOI: $10.3892 /$ ijmm.2021.4921

\begin{abstract}
Circular RNAs (circRNAs) are a class of non-coding RNAs with a circular, covalent structure that lack both $5^{\prime}$ ends and 3 ' poly(A) tails, which are stable and specific molecules that exist in eukaryotic cells and are highly conserved. The role of circRNAs in viral infections is being increasingly acknowledged, since circRNAs have been discovered to be involved in several viral infections (such as hepatitis B virus
\end{abstract}

Correspondence to: Professor Lijun Tang, School of Life Science, Central South University, Xiangya New Campus, Yuelu, 172 Tongzipo Road, Changsha, Hunan 410013, P.R. China

E-mail: tljxie@csu.edu.cn

*Contributed equally

Abbreviations: COVID-19, coronavirus disease 2019; SARS-CoV-2, severe acute respiratory syndrome virus; RNA Pol II, RNA polymerase II; SS, splice site; TER, transcription elongation rate; ICS, intronic complementary sequence; MBNL1, muscleblind like splicing regulator 1; ADAR1, adenosine deaminase 1 acting on RNA1; CDR1, cerebellar degeneration-related protein 1; U1snRNP, U1 small nuclear ribonucleoproteins; IRES, internal ribosome entry site; MBL, muscleblind; EIciRNAs, exon-intron circular RNAs; EIF3J, eukaryotic translation initiation factor 3 subunit J; HBV, hepatitis B virus; HHV, human herpesvirus; HCC, hepatocellular carcinoma; IRF7, interferon regulatory factor 7; cccDNA, hepatitis B virus covalently closed circular DNA; KSHV, Kaposi's sarcoma-associated herpesvirus; PEL, primary exudative lymphoma; vIRF4, viral interferon regulatory factor 4; HSV, herpes simplex virus; EBV, Epstein-Barr virus; HCMV, human cytomegalovirus; pTRS1, protein TRS1; HIV, human immunodeficiency virus; HPV, human papilloma virus; circE7, circRNAs containing the E7 oncogene; MERS-CoV, Middle East respiratory syndrome coronavirus; MAPK, mitogen-activated protein kinase; HTNV, hantavirus; IKK, inhibitors of the nuclear factor $\mathrm{\kappa B}$ kinases; DF, dengue fever; DENV, dengue fever virus; CircPanel, circRNA panel; ROC, receiver operating characteristic; AUC, area under the receiver operating characteristic curve; AFP, $\alpha$-fetoprotein; NPC, nasopharyngeal carcinoma; CC, cervical cancer; Akt, protein kinase B; TNFAIP3, tumor necrosis factor $\alpha$-induced protein 3; WBC, white blood cell; PLT, platelet

Key words: circRNA, viral infection, host cell, biomarker, therapeutic target infection and human papilloma virus infection) through a range of circRNA/microRNA/mRNA regulatory axes. These findings have prompted investigations into the potential of circRNAs as targets for the diagnosis and treatment of viral infection-related diseases. The aim of the present review was to systematically examine and discuss the role of circRNAs in several common viral infections, as well as their potential as diagnostic markers and therapeutic targets.

\section{Contents}

1. Introduction

2. Biogenesis and biological functions of circRNAs

3. Effect of circRNAs on viral infections

4. circRNAs as tools for the diagnosis and prognosis of viral infection-related diseases

5. circRNAs and the treatment of viral infections

6. Conclusions

\section{Introduction}

Circular RNAs (circRNAs/circ) are a group of non-coding RNAs widely found in eukaryotic cells, with a circular and covalently-bonded structure. The majority of circRNAs consist of multiple exons and have been found to be conserved in different species and are specifically expressed during tissue development. Unlike linear RNAs, circRNAs are continuous, covalent structures lacking both the $5^{\prime}$ and $3^{\prime}$ ends, which renders circRNAs stable and resistant to exonuclease-mediated degradation (1). Significant progress has been made of late in circRNA research, which has highlighted the emerging role of circRNAs in cancer, neurological diseases and immune responses (2-4)

The recently acknowledged regulatory role of circRNAs has led to an increasing awareness of their importance in viral infections. The present review summarized the role of circRNAs in frequent viral diseases, with a focus on the possible use of circRNAs for diagnosis and treatment. The latter is particularly relevant in light of the recent emergence of the coronavirus disease 2019 (COVID-19) pandemic caused by the severe acute respiratory syndrome virus (SARS-CoV-2), which is affecting tens of millions of individuals worldwide, due to the very high rate of viral infection (5). 


\section{Biogenesis and biological functions of circRNAs}

Biogenesis. circRNAs are formed by the back-splicing of pre-RNA catalyzed by RNA polymerase II (RNA Pol II). A downstream 5' splice donor site is linked to an upstream 3' splice acceptor site to form a 3'-5' phosphodiester bond that results in a circularized RNA (Fig. 1) (6). Circularization is explained by the 'lariat-driven', 'exon-skipping' and 'intron pairing-driven' models (7). In the lariat model, the partial folding of the RNA during pre-RNA transcription results in non-adjacent exons being pulled close to each other (Fig. 1B). This allows exon jumping to occur, so that the area spanned forms a circRNA intermediate that, by further back splicing, results in circRNA molecules composed of exons. In intron pairing-driven circularization (Fig. 1C), the base-pairing of flanking introns containing complementary sequences (for example, Alu repeats) occurs. This allows the 5' splice site (SS) of a downstream exon sequence to approach the 3' SS of an upstream exon within the intron flanking region, which favors reverse splicing. In addition, a variety of trans-acting protein factors can actively participate in the biogenesis of circRNAs (Fig. 1D).

circRNA expression can be regulated at three levels. First, the transcription of circRNA-producing pre-mRNA occurs via RNA Pol II. It has been found that the average Pol II transcription elongation rate (TER), which is an important factor in determining the outcome of splicing events of nascent circRNA-producing genes, is higher than that of non-circRNA genes (8). It is possible that the TER of a parental gene may influence alternative back-splicing selection. Alternatively, higher processivity might result in less efficient canonical splicing, and then increase back-splicing, Second, it is established that cis and trans-regulatory factors regulate back-splicing during the spliceosome mechanism. The processing of circRNAs can be facilitated by either the RNA pairing of reverse complementary sequences across their flanking introns or protein factors binding to pre-mRNAs to bridge flanking introns together. When the intronic RNA pairing was disrupted by the removal of one intronic complementary sequence (ICS) flanking the circGCN1L1-forming exons by CRISPR/Cas9, circGCN1L1 was completely eradicated in human cells (8). Several RNA-binding proteins (RBPs) have also been reported to play a role in circRNA biogenesis. Muscleblind (MBL) like splicing regulator 1 (MBNL1) was able to bind to and stabilize RNA pairs formed between the ICS, leading to the enhanced circRNA biogenesis. Adenosine deaminase 1 acting on RNA1 (ADAR1) can antagonize circRNA biogenesis by melting the stem structure (8). A recent study also found that the depletion of numerous core spliceosomal components, including the SF3b and SF3a complexes, resulted in preferred circRNA expression, which suggested a shift from canonical splicing to back-splicing under spliceosome starving conditions by depleting spliceosomal factors or chemically inhibiting spliceosomal activities (9). In addition to core spliceosomal factors, a group of splicing regulators are required for circRNA expression in human cells (10). Finally, circRNA turnover may affect circRNA expression. The expression of certain circRNAs can be detected at the steady-state level, largely due to the fact that they can accumulate to high levels post-transcriptionally. A high expression of circRNAs in the brain, possibly due to discrepant decay rates, was observed between circRNAs and their linear counterparts (11).

\section{Biological functions}

MicroRNA (miRNA/miR) sponges. circRNAs are competing endogenous RNAs that can act as miRNA sponges. Through their miRNA response elements, circRNAs can bind to target miRNAs, negatively regulate miRNA expression levels and influence biological activities, thus regulating the expression of target genes (Fig. 1E). For instance, circRNA-7 (ciRS-7) is mainly transcribed from the antisense strand corresponding to cerebellar degeneration-related protein 1 (CDR1), and they always combine with each other as a complex, which is abundant in human and mouse brains. The miR-7 binding sites present in ciRS-7 can adsorb miR-7. A low ciRS-7 level can therefore result in an increase in free miR-7 and the ensuing enhancement of ubiquitin ligase A inhibition $(12,13)$.

$R B P s$. RBPs are mainly involved in the regulation of RNA transcription (14), alternative splicing (15), transport (16) and translation (17). circRNAs can combine RBPs and therefore participate in the regulation of gene expression and protein translation (Fig. 1F). For example, it has been shown that exon-intron circRNAs, such as circ-ETF3 and circ-PAIP2, can bind to U1 small nuclear ribonucleoprotein (U1snRNP) and regulate gene expression in the nuclei of in vitro cultured 293 and HeLa cells (18).

Encoding messengers for transcription. Although circRNAs are non-coding RNAs, the presence of an internal ribosome entry site (IRES) upstream of the circRNA start codon can allow the 40S subunit of eukaryotic ribosomes to bind and initiate translation (Fig. 1G). This was shown by Legnini et al (19), who reported that circ-ZNF609 contains an open reading frame with start and stop codons that can translate into proteins. In addition, Pamudurti et al (20) used ribosome imprinting technology to discover whether circRNA is sufficient to encode proteins.

Competitor of linear RNA. circRNA is the product of RNA processing, thus competition between circRNA and its linear RNA is possible (Fig. 1H). For instance, Ashwal-Fluss's research reported that circMbl is produced by exon 2 of the muscleblind (MBL) gene. The MBL gene product specifically binds to the sequences of circRNA (circMbl) and its flanking introns of the pre-mRNA, and promotes the biosynthesis of circMbl, while circMbl-MBL binding competitively inhibits classical mRNA splicing (21).

Regulating the maternal gene expression. A recent study revealed that circRNAs could regulate the expression of maternal genes at the transcriptional or post-transcriptional level (Fig. 1I) (22). Another study reported a special class of circRNAs, termed Exon-intron circular RNAs (EIciRNAs), which are associated with RNA Pol II in human cells and include circ eukaryotic translation initiation factor 3 subunit J (EIF3J) and circ poly(A) binding protein interacting protein 2 . EIciRNAs are mainly localized in the nucleus, using the 5 ' splicing site of the retained intron to interact with U1 snRNP and improve EIF3J transcription efficiency (23). Thus, EIciRNA can function in transcriptional regulation through RNA-RNA interactions and play different roles from those of circRNAs composed of exonic sequences previously localized in the cytoplasm. 

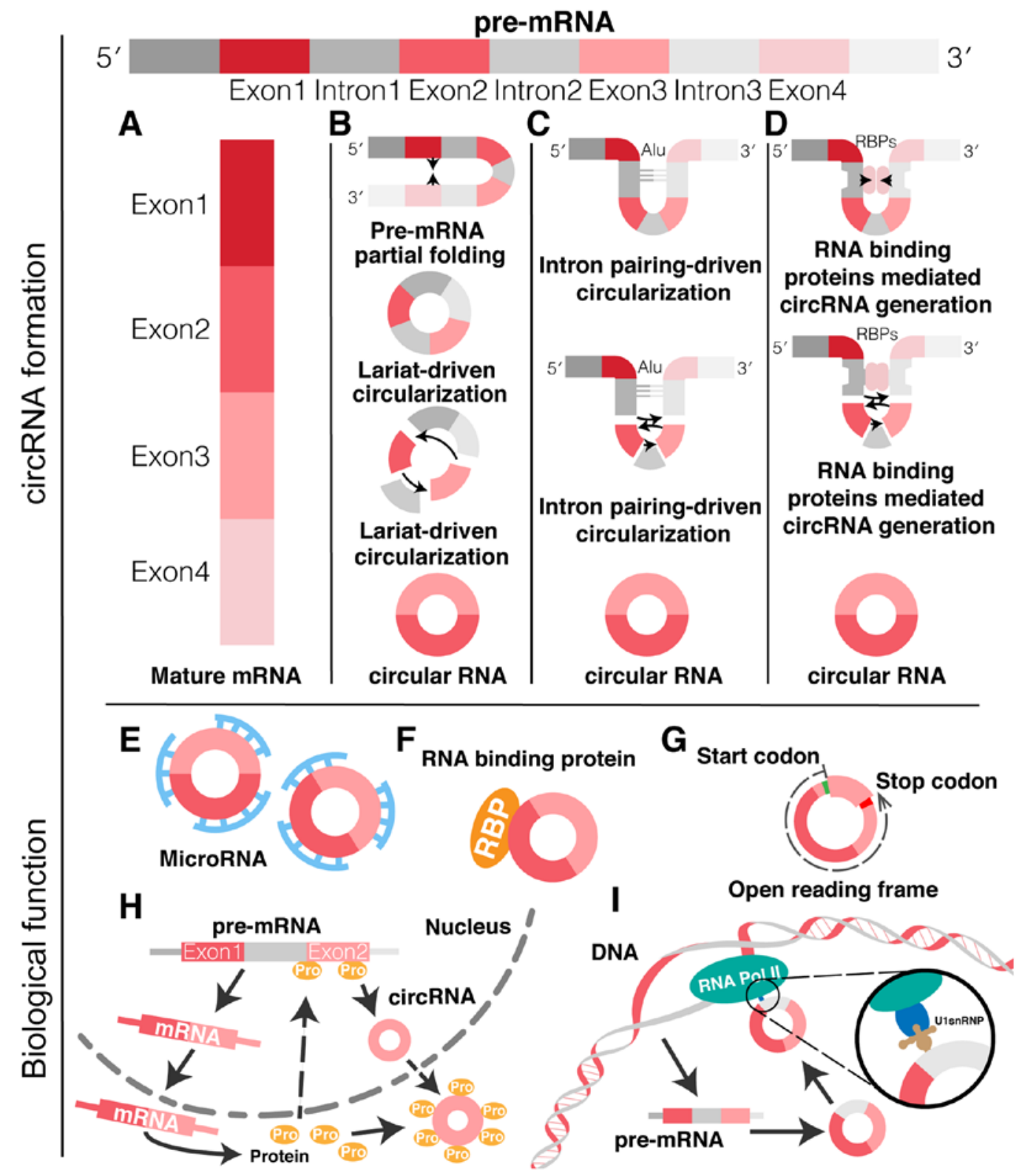

Figure 1. Formation and biological function of circRNAs. (A) Mature mRNA. Generation of circRNAs by back-splicing of pre-RNAs by (B) lariat-driven and (C) intron pairing-driven circularization. (D) The process of circRNA generation can Formation and biological function of circRNAs additionally involve protein regulatory factors. Functions of circRNAs: (E) miRNA sponges, (F) RNA-binding proteins, (G) encoding messengers for transcription, (H) competitors of linear RNA and (I) regulating the expression of maternal genes.

\section{Effect of circRNAs on viral infections}

CircRNAs are involved in the regulation of viral infections, such as hepatitis B virus (HBV) and human herpesvirus (HHV) infections (24). In addition, circRNAs have been proposed as biomarkers to distinguish viral from non-viral pneumonia (25). The crucial role of circRNAs in viral infections is associated with controlling miRNA levels and regulating innate immune responses (Table I).

$H B V$. HBV is a partially double-stranded DNA virus of $\sim 3,200$ base pairs (26). Infections by HBV are among the most common chronic viral diseases and can result in the later development of cirrhosis or hepatocellular carcinoma (HCC). The prevalence of hepatitis B surface antigen ( $\mathrm{HBsAg}$ ) seropositivity has been reported to be
$3.61 \%$ worldwide, with a $95 \%$ confidence interval (27). The expression levels of host circRNAs can be affected by HBV infections. Research on the differential expression of RNAs following HBV infection has indicated the involvement of four circRNA/miRNA/mRNA pathways. Both the hsa circ_0005389-miR-4505/miR-6752-5p/miR-5787-interferon regulatory factor 7 (IRF7) and hsa_circ_0000038-miR-370/ miR-939/HBV pathways are significantly upregulated, while the hsa_circ_0000650/miR-6873-3p/transforming growth factor (TGF) $\beta$ and hsa_circ_0000650/miR-210-5p/HBV pathways are downregulated (28). Studies on the downstream genes of these four pathways have shown that circRNAs are involved in HBV replication, as well as in the liver inflammation and fibrosis caused by HBV infection. hsa_circ_0005389 and hsa_circ_0000038 are associated with HBV replication. IRF7 of the 0005389 pathway is involved in interferon 
Table I. Roles of circRNAs in viral infections.

\begin{tabular}{|c|c|c|c|c|}
\hline Virus & Diseases or infections & circRNA & Role & (Refs \\
\hline \multirow[t]{10}{*}{ HBV } & HBV-related hepatitis & hsa_circ_0005389 & $\begin{array}{l}\text { Associated with the transcription of viral } \\
\text { cccDNA and HBV replication in hepatocytes. }\end{array}$ & $(29)$ \\
\hline & & hsa_circ_0000038 & $\begin{array}{l}\text { Associated with HBV Enhancer I, HBV } \\
\text { enhancer II and core promoter. }\end{array}$ & $(31)$ \\
\hline & Liver fibrosis & hsa_circ_0000650 & $\begin{array}{l}\text { Involved in liver fibrosis through the hsa_ } \\
\text { circ_0000650-miR-6873-3p-TGF } \beta \text { pathway. }\end{array}$ & (34) \\
\hline & HBV infection & hsa_circ_0000650 & $\begin{array}{l}\text { Interacts with miR-210, which binds to the } \\
\text { pre-S1 antigenic domain of the HBV envelope } \\
\text { protein (HBsAg). }\end{array}$ & $(35)$ \\
\hline & HBV-related carcinoma & hsa_circ_00004812 & $\begin{array}{l}\text { Associated with the oncogenesis induced by } \\
\text { HBV infection through circ_00004812-miR- } \\
\text { 1287-5p-FSTL1 pathway. }\end{array}$ & $(37)$ \\
\hline & & hsa_circ_4099 & $\begin{array}{l}\text { Associated with HBV infection and liver } \\
\text { fibrosis. }\end{array}$ & $(39)$ \\
\hline & & hsa_circ_0000976 & Differentially expressed in HBV-related HCC. & $(41)$ \\
\hline & & hsa_circ_0007750 & $\begin{array}{l}\text { Associated with chemokine- and } \\
\text { cytokine-mediated immune responses. }\end{array}$ & $(42)$ \\
\hline & & hsa_circ_0027089 & Promote HBV replication. & $(43)$ \\
\hline & & hsa_circ_100338 & $\begin{array}{l}\text { Correlated with low survival and metastatic } \\
\text { progression. }\end{array}$ & $(45)$ \\
\hline KSHV & KSHV infection & hsa_circ_0001400 & Inhibiting the expression of certain viral genes. & $(50)$ \\
\hline HSV & HSV-1 infection & circRNAs & $\begin{array}{l}\text { Implicated in signaling and apoptosis } \\
\text { pathways, the cell cycle and cell death. }\end{array}$ & $(53)$ \\
\hline \multirow[t]{2}{*}{ EBV } & EBV infection & ebv-circRPMS 1 & $\begin{array}{l}\text { A novel regulation agent acting on } \\
\text { transcription and/or interfering with splicing } \\
\text { as a miRNA sponge. }\end{array}$ & $(54)$ \\
\hline & EBV-related tumor & $\begin{array}{l}\text { circLMP2, } \operatorname{circBHLF} 1 \\
\text { circBARTs }\end{array}$ & $\begin{array}{l}\text { Associated with proliferation of tumor cells of } \\
\text { EBV-positive patients. }\end{array}$ & $(48)$ \\
\hline HCMV & HCMV infection & $\begin{array}{l}\text { hsa_circ_0001445 } \\
\text { hsa_circ_0001206 }\end{array}$ & Participated in a HCMV latency mechanism. & $(59)$ \\
\hline HIV & HIV infection & circRNAs & $\begin{array}{l}\text { Associated with HIV replication and T cell } \\
\text { infection. }\end{array}$ & $(60)$ \\
\hline HPV & $\begin{array}{l}\text { HPV-related cervical } \\
\text { cancer }\end{array}$ & HPV-circE7 & $\begin{array}{l}\text { Essential for the transformation and growth } \\
\text { of cervical cancer cells, also promote virus } \\
\text { replication and host cell transformation. }\end{array}$ & $(64)$ \\
\hline MERS-CoV & MERS-CoV infection & $\begin{array}{l}\text { circFNDC3B } \\
\text { circCNOT1 }\end{array}$ & $\begin{array}{l}\text { Associated with the regulation of MERS-CoV } \\
\text { cellular proliferation. }\end{array}$ & $(67)$ \\
\hline HTNV & HTNV infection & $\begin{array}{l}\operatorname{circ} 0002470 \\
\operatorname{circ} 0006132 \\
\operatorname{circ0000479}\end{array}$ & Associated with HTNV infection. & $(71)$ \\
\hline SARS-CoV-2 & SARS-CoV-2 infection & $\begin{array}{l}\text { Ppp1r10 } \\
\text { C330019G07Rik }\end{array}$ & Related with SARS-CoV-2 infection. & (77) \\
\hline IAV & Influenza virus infection & circ-GATAD2A & In association with viral replication. & $(79)$ \\
\hline EBOV & EBOV infection & circRNA_chr19 & $\begin{array}{l}\text { May have effect on EBOV infection through } \\
\text { the circRNA_chr19-miR-30b-3p-CLDN18 } \\
\text { pathway. }\end{array}$ & $(80)$ \\
\hline DENV & Dengue fever & $\begin{array}{l}\text { hsa_circ_0006459 } \\
\text { hsa_circ_0015962 }\end{array}$ & $\begin{array}{l}\text { Associated with reductions in white blood cell } \\
\text { (WBC) and platelet (PLT) counts. }\end{array}$ & $(84)$ \\
\hline
\end{tabular}


(IFN)- $\alpha$-mediated antiviral immunity (29). IFN- $\alpha$ reduces the acetylation level of histone $\mathrm{H} 4$ bound to HBV covalently closed circular DNA (cccDNA), which inhibits the transcription of viral cccDNA and HBV replication in hepatocytes (30). miR-370 of the 0000038 pathway restrains HBV gene expression by targeting the 3'-untranslated region of the transcription factor nuclear factor IA (NFIA) mRNA, with NFIA being able to regulate the activity of HBV enhancer I (31). miR-939, also of the 0000038 pathway, suppresses HBV replication by reducing the activity of the HBV enhancer II/core promoter (32).

TGF $\beta$ inhibits the anti-fibrotic function of natural killer cells in liver fibrosis; therefore, hsa_circ_0000650 may be involved in liver fibrosis through the hsa_circ 0000650/miR-6873-3p/TGFß signaling pathway (33). circRNA hsa_circ_0000650 also plays a role in inflammation through the hsa_circ_0000650/miR-210-5p/HBV pathway. In fact, inflammation results in liver hypoxia, which leads to increases in liver and serum miR-210 levels (34). In addition, bioinformatics analysis has indicated that miR-210 binds to the HBV pre-S1 region and reduce HBsAg expression, which leads to inhibition of hepatitis B virus replication (35). However, no significant correlation between miR-210 and HBsAg levels has been proven by experiments (36), casting doubts on such an association.

Other circRNA/miRNA/mRNA pathways have also been associated with HBV infectivity. The expression level of circ_00004812 [circ_00004812/miR-1287-5p/follistatin like 1 (FSTL1) pathway] has been found to be increased in HBV-infected cells. The knockdown of circ_00004812 has been shown to lead to an increase in the mRNA and protein expression levels of IFN- $\alpha$ and IFN- $\beta$ (37). FSTL1 and IFN- $\beta$ have been reported to participate in the oncogenesis process induced by HBV infection (38). With regards to the association between HBV infection and liver fibrosis, hsa_circ_4099 enhances $\mathrm{H}_{2} \mathrm{O}_{2}$-mediated fibrosis, as well as exerts a sponge effect on miR-706 (39). miR-706 is known to inhibit oxidative stress-induced fibrosis in hepatocytes (40). Furthermore, the expression levels of circRNAs have been reported to be altered in the plasma of HBV-related patients with cancer, where hsa_circ_0000976 and hsa_circ_0007750 were also found to be differentially expressed (41). According to the database Circular RNA Interactome, hsa_circ_0000976 has a binding site for hsa-miR-486-3p. The latter is significantly upregulated in infected hepatocytes and is associated with chemokine- and cytokine-mediated immune responses (42). hsa_circ_0027089 is another circRNA upregulated in the plasma of patients suffering from HBV-related HCC. It has a binding site for hsa-miR-15b-3p and hsa-miR-141-5p (43). miR-141 downregulates the peroxisome proliferator-activated receptor $\alpha$ and inhibits HBV replication by reducing the HBV promotor activity (44). The upregulation of circRNA_100338 of the circRNA-100338/miR-141-3p pathway is closely correlated with a low survival and metastatic progression in HBV-related $\mathrm{HCC}$ and has indeed been suggested as a biomarker of disease progression (45). Not only can HBV induce changes in host circRNA levels, but it can also be the source of circRNAs of viral origin that arise during its replication. The generation of viral circRNAs is under the influence of host factors, as demonstrated by the observation that knocking down the host protein DexH-box helicase 9 results in an increase in circRNAs of viral origin (46).
$H H V$. A number of circRNAs have been suggested to participate in herpes virus infections by acting as miRNA sponges, as well as by encoding proteins. These circRNAs appear to affect the host's cellular environment by linking the viral behavior to pathogenic mechanisms (47).

Kaposi's sarcoma-associated herpesvirus (KSHV)/HHV8. KSHV/HHV8 infection can result in Kaposi's sarcoma, endothelium-derived angiogenesis-induced tumors, primary exudative lymphomas (PEL) and multicenter Castleman disease. KSHV-coding circRNAs are mostly derived from the viral interferon regulatory factor 4 (vIRF4)/K10 site, the polyadenylated nuclear site and the K7.3 site (48-50). Abere et al (51) determined the expression characteristics of circ-vIRF4, circPANs and circK7.3s in PEL cell lines, tumor tissues and patients' sera. Varying degrees of expression of KSHV-encoded circRNAs were commonly observed in PEL cells. These circRNAs are easy to detect because they are packaged into particles. Some of the circRNAs generated during KSHV infection possess miRNA binding sites, such as circRNA hsa_circ_0001400, which can inhibit the expression of certain viral genes (50). It has also been reported that host circRNAs can be exported from infected cells in extracellular vesicles acting as vectors that deliver antiviral signals to surrounding cells (52). On the whole, it is known that the production of antiviral circRNAs is activated by KSHV infection, with their expression being induced during the lytic phase of infection.

Herpes simplex virus $(H S V-1) / H H V 1$. HSV-1/HHV1 is a member of the herpesvirus and $\alpha$ herpesvirus subfamilies, which feature double-stranded linear DNA genomes. HSV-1 infection in human lung fibroblast cell lines results in a vast number of circRNAs, genes and miRNAs being involved in responses to the viral infection along the circRNA-miRNA-gene regulatory axis. Such genes are implicated in signaling and apoptosis pathways, the cell cycle and cell death (53).

Epstein-Barr virus $(E B V) / H H V 4$. EBV/HHV4 is a virus from the lymphotropic genus of the herpesvirus family with linear, double-stranded DNA. It can cause a variety of human lymphoid and non-lymphoid malignancies and is also associated with nasopharyngeal and gastric cancer. Huang et al (54) identified the EBV-encoded circRNA ebv-circRPMS1 in the cytoplasm and nucleus of EBV-infected cell lines, which has been regarded as a novel regulatory agent acting on transcription and/or interfering with splicing as an miRNA sponge. The following EBV-encoded circRNAs have been identified to be associated with oncogenes: Circ proteasome $20 \mathrm{~S}$ subunit $\beta 9$ (LMP2), circBHLF1 and circ barttin CLCNK type accessory subunit $\beta$. The latter has been shown to play a role in the proliferation of tumor cells of EBV-positive patients (48). Host miRNAs, such as miR-15b-5p, miR-30c-1-3p, miR-30c-2-3p, miR-424-5p and miR-4286, have been recognized as targets of EBV circRNAs. These miRNAs, which are strongly linked to herpes infections, have been found to also be linked to tumorigenesis and tumor invasion (55-57).

Human cytomegalovirus (HCMV)/HHV5. The HCMV/ HHV5 protein TRS1 (pTRS1) has been shown to enhance the activity of the IRES through its binding to a circRNA reporter. The expression of pTRS1 also increases the activity of IRESs, which controls the expression of proteins needed for efficient HCMV replication within the cell (58). This indicates 
that circRNAs can play a relevant role in HCMV infections. Lou et al (59) reported the downregulation of the circRNAs hsa_circ_0001445 and hsa_circ_0001206 in latently infected THP-1 monocyte-macrophage cell line and patients infected with HCMV compared with normal controls. These differentially expressed circRNAs can bind to miRNAs such as hsa-miR-21, therefore participating in a HCMV latency mechanism.

Human immunodeficiency virus (HIV). HIV, widely known as the cause of the acquired immune deficiency syndrome, is a retrovirus. Three series of circRNAs have been reported to be involved in three different regulatory pathways in the early stages of HIV infection: circRNA-miR-542-3p/ miR-101-3p/let-7c-5p-CDKN1A, circRNA/miR-27b-3p/ CCNK and circRNA/miR-548ah-3p/interleukin (IL)-15 (60). CDKN1A encodes the cyclin-dependent kinase inhibitor 1 that inhibits HIV replication (61). Cyclin K, encoded by CCNK, inhibits HIV gene expression and replication by using HIV-1 Nef protein to displace Cyclin-T1 from the positive elongation factor b complex (62). IL-15 facilitates $\mathrm{CD} 4^{+} \mathrm{T}$ cell infection, therefore creating an HIV reservoir (63).

Human papilloma virus (HPV). HPV is a small double-stranded DNA virus that infects stratified epithelial cells. The majority of HPV infections are asymptomatic or cause benign wart growth, but some high-risk HPVs can promote the development of cervical and oropharyngeal cancer. Zhao et al (64) observed that HPVs can generate circRNAs containing the E7 oncogene (circE7), which encodes the E7 oncoprotein. HPV16 circE7 is essential for the transformation and growth of CaSki cervical cancer (CC) cells. circE7 may also promote virus replication and host cell transformation, suggesting a link between circE7 and high-risk HPV, and making circE7 abundance of prognostic value.

Middle East respiratory syndrome coronavirus (MERS-CoV). MERS-CoV is a coronavirus that affects the human respiratory system by causing fever, chills and severe dyspnea (65). The pathogenesis of this virus is not yet fully understood (66). Recent research has reported that the knockdown of circ fibronectin type II domain containing 3B (FNDC3B) and circ CCR4-NOT transcription complex subunit 1 (CNOT1) with specific small interfering RNAs (siRNAs) reduces the viral load of infected cells and their target mRNA expression in human Calu-3 lung adenocarcinoma epithelial cells, resulting in a reduction in the expression of image result for map $3 \mathrm{k} 9$ definition (MAP3K9) (67). MAP3K9 is an upstream modulator of the MAPK signaling pathway that is involved in cell proliferation (68), as well as in MERS-CoV infection (69). Therefore, circRNAs may play an important role in the regulation of MERS-CoV cellular proliferation, and could prove useful for the development of novel antiviral strategies.

In addition, it has been found that the knockdown of circFNDC3B and circCNOT1 significantly reduce the MERS-CoV viral load in Calu-3 and HFL cells, which may be associated with the downregulation of target genes regulated by circFNDC3B and circCNOT1 (67). MAP3K9 is an upstream modulator of the MAPK pathway that can affect many aspects of cell proliferation, migration and apoptosis (68), while
siRNA knockdown of circFND3B or circCNOT1 leads to a significant decrease in the expression of MAP3K9. This may provide novel insight into the modulation of the extracellular signal-regulated kinase (ERK)/MAPK pathway as a host-targeted antiviral strategy against MERS-CoV infection.

Hantavirus (HTNV). HTNV is a zoonotic virus that infects humans and leads to hemorrhagic fever with renal syndrome, hantavirus pulmonary syndrome or hantavirus cardiopulmonary syndrome (70). In total, 4 circRNA/miRNA/mRNA regulatory axes have been found to be involved in HTNV infections by ceRNA analysis: circ0002470/miR-3182/ interferon induced protein 44 (IFI44), circ0006132/miR1304-3p/2'-5'-oligoaenylate synthetase 1 (OAS1), circ0000479/ miR-149-5p/retinoic acid-inducible gene I (RIG-I) and circ0000479/miR-337-3p/caspase 1 (CASP1) (71).

IFI44 is an IFN-inducible protein associated with viral infections. It can rescue the inhibition of IFN-induced viral growth. More precisely, it inhibits the promoter activity of HIV-1 LTR, thereby affecting viral transcription (72). Furthermore, IFI44 interacts with the immunoregulatory protein FK506-binding protein 5, which binds to cellular kinases that are inhibitors of the nuclear factor $\kappa \mathrm{B}$ kinases (IKK) $\alpha$, IKK $\beta$ and IKK $\varepsilon$. Such inhibition results in a negative modulation of IFN responses (73). The antiviral protein, OAS1, is induced by viral infection [Sendai or influenza A virus (IAV)], an induction that requires a type I IFN response (74). RIG-I is a pattern recognition receptor that, upon recognition of RNA viruses, activates the expression of type I interferons, hence promoting an antiviral response (75). CASP1 is a component of the inflammasome, a complex involved in the activation of the pro-inflammatory cytokines IL-18 and IL-1 $\beta$ that lead to inflammatory cell death (76).

In brief, circRNAs may provide new targets for interventions aimed at controlling HTNV infections.

$S A R S-C o V-2$. In the case of SARS-CoV-2 infection, one miRNA (miR-124-3p), one lncRNA (Gm26917), one transcription factor (STAT2), one mRNA (DeXD/H-box helicase 58) and two circRNAs (protein phosphatase 1 regulatory subunit 10 and C330019G07Rik) have been found to comprise a quintuple network (77). miR-124-3p has been confirmed to be upregulated in the case of HIV-1 infection, resulting in the downregulation of p21 and potassium two pore domain channel subfamily $\mathrm{K}$ member, which leads to increased virion release (78). However, the main targets of this network remain unclear, and an in-depth study of the release of certain inflammatory mediators may provide new insight into the diagnosis of SARS-CoV-2 infection.

Other viral infections. circRNAs have also been found to be linked to several other viral infections as major regulatory molecules of signaling pathways, and even targets for disease diagnosis and treatment.

$I A V$. The infection of A549 human alveolar basal epithelial cells with IAV H1N1 promotes the formation of high levels of circ-GATA zinc finger domain containing 2A (GATAD2A). In parallel, circ-GATAD2A knockdown enhances autophagy, as established by determining LC3-II and p62 levels, while it inhibits H1N1 replication. Conversely, circ-GATAD2A 
Table II. circRNAs as tools for the diagnosis and prognosis of viral infection-related diseases.

\begin{tabular}{ll} 
Diseases & \\
\hline HBV-related & hsa_circ_0000976 \\
hepatocellular & hsa_circ_0007750 \\
carcinoma & hsa_circ_0139897
\end{tabular}

Diagnostic indicators

(Refs.)

The plasma circRNA panel (CircPanel) containing hsa_circ_0000976, hsa_circ_0007750 and hsa_circ_0139897 is used to detect the changes of circRNA contents in plasma for clinical diagnosis of HBV-related hepatocellular carcinoma (HCC). This method performs better than alpha-fetoprotein (AFP) in the diagnosis of small-HCC and could also identify AFP-negative HCC and AFP-negative small-HCC.

$\begin{array}{lll}\begin{array}{l}\text { EBV-related } \\ \text { nasopharyngeal }\end{array} & \text { circRPMS1 } & \begin{array}{l}\text { CircRPMS1 was found to be significantly increased in EBV-positive } \\ \text { NPC tissues compared with adjacent tissues, while circRPMS1 was } \\ \text { not expressed in EBV-negative NPC tissues. }\end{array} \\ & \begin{array}{l}\text { hsa_circRNA_001387 } \\ \text { In EBV infection, hsa_circRNA_001387 is significantly upregulated. } \\ \text { HPV-positive }\end{array} & \begin{array}{l}\text { circE7 } \\ \text { circE7 as a possible sensitive marker for high-risk HPV. }\end{array}\end{array}$

cervical cancers

HBV, hepatitis B virus; EBV, Epstein-Barr virus; HPV, human papilloma virus.

overexpression impairs autophagy and favors H1N1 replication. The enhancement of viral replication is thought to be due to the interaction of circ-GATAD2A with the vacuolar protein sorting 34, since the knockdown of the latter blocks autophagy and results in an increase of $\mathrm{H} 1 \mathrm{~N} 1$ replication. In conclusion, circ-GATAD2A may serve as a potential therapeutic target in connection with influenza virus infections (79).

Ebola virus. In Ebola virus infection, circRNA_chr19 regulates the expression of claudin 18 (CLDN18) by targeting miR-30b-3p, thus reducing the inhibitory effect of miR-30b-3p on CLDN18 through the sponge effect (80). CLDN18 is likely to be involved in preserving epithelial integrity (81), with its effect on Ebola virus infections requiring further research.

Dengue fever $(D F)$ virus $(D E N V)$. DF is an acute infectious disease caused by Aedes aegypti mosquito-transmitted DENV. The incidence of DF has increased substantially worldwide, with its clinical manifestations including acute attacks, sudden high fever, fatigue, aches, loss of appetite and nausea (82). The main diagnostic tool of DF is the reduction in white blood cell (WBC) and platelet (PLT) counts (83). circRNA hsa_circ_0006459 levels have been found to be negatively associated with WBC, PLT and lymphocyte counts in the peripheral blood of patients with DF, while hsa_circ_0015962 levels have been found to be positively associated with the same parameters. With regards to the mechanisms of action, hsa_circ_0015962 binds to miR-4683 and hsa_circ_0006459 binds to miR-133b. The levels of these circRNAs may thus be considered as potential biomarkers (84).

\section{4. circRNAs as tools for the diagnosis and prognosis of viral infection-related diseases}

The rapid development of high-throughput sequencing and bioinformatics in recent years has allowed for the detection of circRNAs highly expressed in virus infection-related diseases. The fact that circRNAs are abundant, ubiquitous, stable, conserved across species and tissue-cell specific indicates a diagnostic potential greater than that of linear RNA (85).
circRNAs can therefore serve as novel diagnostic biomarkers of viral infections (Table II).

Recent research has demonstrated that a plasma circRNA panel (CircPanel) containing hsa_circ_0000976, hsa_circ_0007750 and hsa_circ_0139897 could be used as a biomarker for the clinical diagnosis of HBV-related hepatocellular carcinoma. This HCC detection CircPanel was constructed based on microarray screening and reverse transcription-quantitative PCR to identify plasma circRNAs that are increased in patients with HCC with HBV compared with the controls. The diagnostic accuracy of the method was evaluated by constructing a logistic regression model and analyzing the area under the receiver operating characteristic (ROC) curve (AUC). This CircPanel performed better than $\alpha$-fetoprotein (AFP) in the diagnosis of small HCC and could also identify AFP-negative HCC and AFP-negative small HCC (41).

Nasopharyngeal carcinoma (NPC), mainly induced by $\mathrm{EBV}$, is one of the most difficult cancers to detect. Thus, specific biomarkers of NPC are very helpful for a timely diagnosis of NPC. A recent study reported that circRPMS1 was found to be significantly increased in EBV-positive NPC tissues compared with adjacent tissues, and was not expressed in EBV-negative NPC tissues, suggesting that circRPMS1 could indeed be a useful NPC diagnostic biomarker (86). In addition, a marked upregulation of hsa_circRNA_001387, which could be used as a novel NPC biomarker, has been reported. The ROC curve for the determination of the diagnostic value of hsa_circRNA_001387 showed a high accuracy, indicating that this circRNA could be a specific and sensitive NPC marker (87).

circE7 is present in RNA-Seq data from The Cancer Genome Atlas concerning HPV-positive cancers and cell lines with only episomal HPVs. Given the stability of circRNA and the importance of the E7 oncoprotein in the genesis of $\mathrm{CC}$, it has been considered appropriate to examine circE7 as a possible, sensitive marker of high-risk HPVs (64). Zhou et al (88) examined the diagnostic potential of ciRS-7 regarding $\mathrm{CC}$ by assessing $\mathrm{ROC}$ curves, and concluded that it is indeed an indicator of the activity of CC cells. 
Table III. circRNAs and the treatment of viral infections.

\begin{tabular}{|c|c|c|c|}
\hline circRNA & Role in viral infection & Therapeutic potential & (Refs.) \\
\hline circ-10156 & $\begin{array}{l}\text { Acting as a molecular sponge for } \\
\text { miR-149-3p, regulating the proliferation } \\
\text { of HBV-related liver cancer cells through } \\
\text { the miR-149-3p/Akt1 pathway. }\end{array}$ & $\begin{array}{l}\text { It was observed that circ- } 10156 \text { was } \\
\text { up-regulated in liver cancer tissues, and } \\
\text { inhibition of circ- } 10156 \text { expression in liver } \\
\text { cancer tissues could inhibit the proliferation } \\
\text { of liver cancer cells. }\end{array}$ & $(89-91)$ \\
\hline circ-ATP5H & $\begin{array}{l}\text { Acts as miR-138-5p sponge and regulates } \\
\text { the expression of TNFAIP3. }\end{array}$ & $\begin{array}{l}\text { Inhibit the expression of circ-ATP5H, and } \\
\text { enhance the anticancer effect of miR-138-5p. }\end{array}$ & (92) \\
\hline circ-0004812 & $\begin{array}{l}\text { By inhibiting miR-1287-5p, it promotes } \\
\text { the expression of FSTL1, thereby } \\
\text { regulating the immune suppression } \\
\text { induced by HBV. }\end{array}$ & $\begin{array}{l}\text { Releasing the inhibitory effect of } \\
\text { circ-0004812, thereby inhibiting the } \\
\text { expression of FSTL1, and inhibiting its HCC } \\
\text { cell proliferation promotion and apoptosis } \\
\text { inhibitory effect. }\end{array}$ & $(37)$ \\
\hline circ-RPMS1 & $\begin{array}{l}\text { Sponge multiple miRNAs (miR-203, } \\
\text { miR-31 and miR-451) and promote } \\
\text { epithelial-mesenchymal transition } \\
\text { to promote the occurrence of } \\
\text { nasopharyngeal carcinoma. }\end{array}$ & $\begin{array}{l}\text { Reduce or eliminate the sponge } \\
\text { effect of circRPMS1 and inhibit the } \\
\text { epithelial-mesenchymal transition of } \\
\text { nasopharyngeal carcinoma. }\end{array}$ & $(86,93,94)$ \\
\hline $\begin{array}{l}\text { circ-LMP2A } \\
\text { (from } \mathrm{EBV} \text { ) }\end{array}$ & $\begin{array}{l}\text { Attenuate the tumor suppressor effect of } \\
\text { miR-3908/TRIM59/p53 axis to promote } \\
\text { EBV-related gastric cancer cell metastasis } \\
\text { and tumor progression. }\end{array}$ & $\begin{array}{l}\text { Inhibit circ-LMP2A derived from EBV and } \\
\text { strengthen the tumor suppressive effect of } \\
\text { miR-3908/TRIM59/p53 axis. }\end{array}$ & (96) \\
\hline $\begin{array}{l}\text { circ-FNDC3B } \\
\text { and circ-CNOT1 }\end{array}$ & $\begin{array}{l}\text { Associated with the MERS-CoV load in } \\
\text { Mers-CoV-infected lung adenocarcinoma } \\
\text { and its target mRNA expression, and } \\
\text { significantly affect mitogen-activated } \\
\text { protein kinase (MAPK) and } \\
\text { ubiquitination pathway. }\end{array}$ & $\begin{array}{l}\text { Knockout of circFNDC3B and circCNOT1 } \\
\text { will result in decreased cell virus content. }\end{array}$ & $(67)$ \\
\hline $\begin{array}{l}\text { Artificial circRNA } \\
\text { sponge }\end{array}$ & Artificially synthesized circRNA & $\begin{array}{l}\text { Isolate miR-122, thereby inhibiting the } \\
\text { production of viral proteins in the HCV cell } \\
\text { culture system. }\end{array}$ & (97) \\
\hline
\end{tabular}

circRNA, circular RNA; HBV, hepatitis B virus; TNFAIP3, tumor necrosis factor $\alpha$-induced protein 3; EBV, Epstein-Barr virus; TRIM59, tripartite motif containing 59; MERS-CoV, Middle East respiratory syndrome coronavirus.

The altered expression of circRNAs is expected to become an important molecular tool for the early diagnosis of viral infection-related diseases, which can result in an increased patient long-term survival, reducing the mortality rate and improving prognosis.

\section{5. circRNAs and the treatment of viral infections}

circRNAs can also be used as therapeutic targets in the management of viral infections (Table III). circRNA-10156 is abundantly expressed in HBV-related liver cancer and can regulate the proliferation of malignant cells through the circRNA_10156/miR-149-3p/AKT1 signaling pathway (89). The depletion of circRNA_10156 can increase the content of miR-149-3p and decrease AKT1 levels. AKT1 mediates the epithelial-mesenchymal conversion of HCC cells through the AKT/glycogen synthase kinase- $3 \beta / \beta$-catenin signaling pathway and inhibits HBV replication by suppressing gene transcription $(90,91)$. In addition, the increased expression of hsa_circ_0006942 (circ-ATP5H) and tumor necrosis factor $\alpha$-induced protein 3 (TNFAIP3) were observed in HCC cells associated with HBV infection, while the expression of miR-138-5p was downregulated. Further experiments proved that circ-ATP synthase peripheral stalk subunit $\mathrm{d}$ (ATP5H) acts as an miR-138-5p sponge and regulates the expression of TNFAIP3, thus circ-ATP5H may be a potential therapeutic target for HBV-related HCC (92). The circ_0004812/miR-1287-5p/FSTL1 pathway regulates HBV-induced immune system suppression (37). circ_0004812 inhibits miR-1287-5p, which promotes FSTL1 expression leading to the expansion of HCC cells, an increase in cellular proliferation and the inhibition of cellular apoptosis. EBV $(+)$ NPC tissues express high levels of circRPMS1. circRPMS1 knockdown can reduce the invasiveness of NPC cells. Cell invasion is mediated by miR-203, miR-31 and miR-451, as shown by the fact that inhibitors of these miRNAs can reverse 


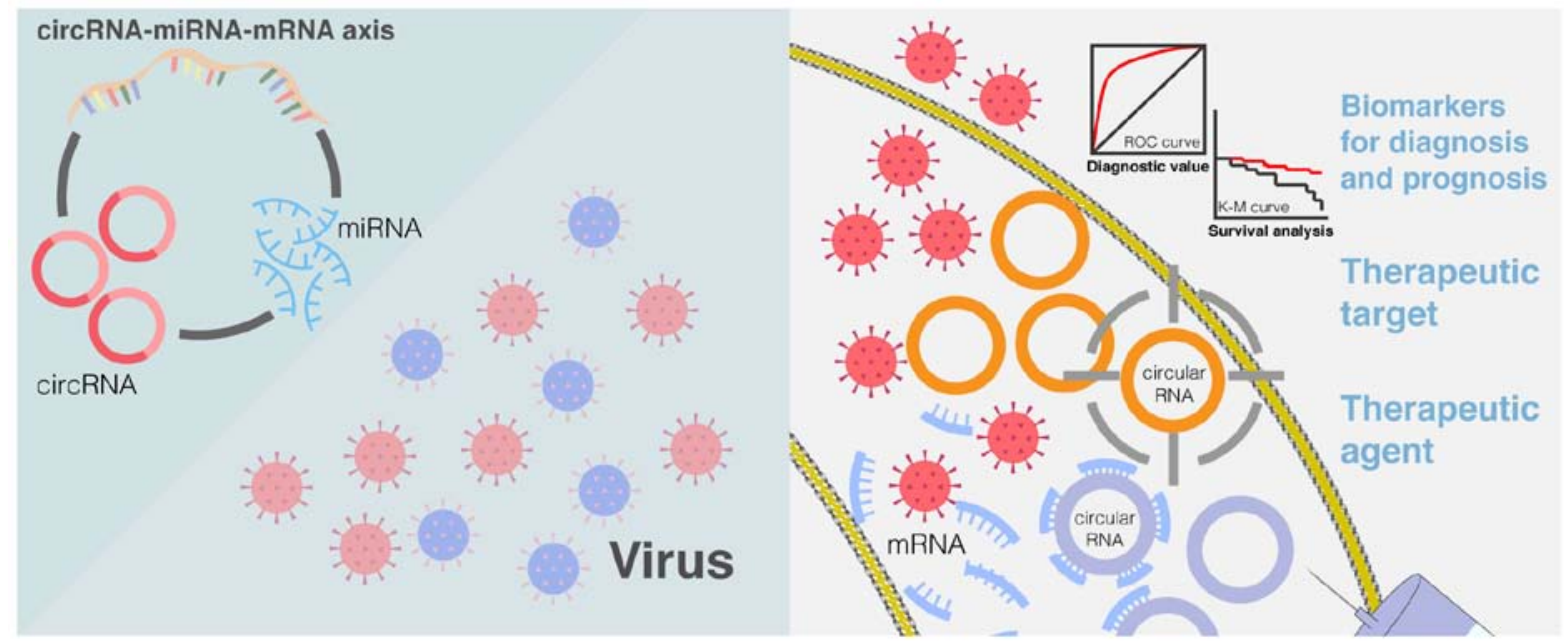

Figure 2. Effects and applications of circRNAs on viral infection. A series of circRNA/microRNA/mRNA regulatory axes are involved in viral infections. Based on the specific circRNA in viral infection, circRNAs have the potential for use as targets for the diagnosis and treatment of viral infection-related diseases.

the circRPMS1 knockdown effects. circRPMS1 can sponge the aforementioned miRNAs, therefore mediating NPC oncogenesis, which occurs by promoting epithelial-mesenchymal transition. As a consequence, circRPMS1 can likely become a convenient therapeutic target for the treatment of EBV-associated NPC $(86,93,94)$.

circRNAs have been found to be involved in EBV-associated gastric cancer. In fact, circRPMS1_E4_E3a and circRPMS1_ E4_E2 were detected in EBV-positive stomach cancer patient specimens (95). In a recent study, the expression of the EBV-encoded ebv-circLMP2A was found to be associated with the induction of stem cell-like phenotypes in a tumor cell line through targeting the miR-3908/tripartite motif containing 59 (TRIM59)/p53 axis. Of note, a high expression of ebv-circLMP2A has been found to be significantly associated with metastasis and a poor prognosis of patients with gastric cancer, indicating the potential of this EBV-encoded circRNA as a therapeutic target (96).

The expression of hsa_circ_0067985/circFNDC3B and hsa_circ_0006275/circCNOT1 has been found to be upregulated in MERS-CoV-infected human lung adenocarcinoma epithelial cells. The knockdown of circFNDC3B and circCNOT1 results in a reduction of both cellular virus content and the expression of the target mRNAs, which modulate MAPK and ubiquitination pathways. These results provided insights into potential antiviral strategies for MERS-CoV infections (67).

circRNAs can be engineered as miRNA sponges. In a model system, advantage was taken of the fact that the life cycle of HCV relies on host miR-122 (97). Artificial circRNA sponges were devised to sequester miR-122, which resulted in the inhibition of $\mathrm{HCV}$ production in cell culture systems. These findings uncovered the potential application of circRNAs to tackle HCV infection (98).

\section{Conclusions}

circRNAs are specific non-coding RNAs involved in the regulation of miRNAs, and thus affect gene expression. These RNAs titrate miRNAs and regulate transcription and/or interfere with splicing. The regulatory role of circRNAs in the immunity to viral infections has received increasing attention. The analysis of the specific mechanisms through which circRNAs can influence viral infections would facilitate a deeper understanding of disease etiologies. In addition, such studies will likely unveil novel therapeutic targets and possible treatments for infectious diseases that involve changes in circRNA levels (Fig. 2), including COVID-19. These new approaches are expected to lead to improved prognoses of viral diseases.

\section{Acknowledgements}

The authors would like to thank Dr Rodolfo Garcia (Biomedicine Research Honorary Consultant, London, United Kingdom) for commenting on and revising the manuscript.

\section{Funding}

The present study was supported by the Changsha Municipal Natural Science Foundation (grant no. kq2007065), Teacher Research Fund of Central South University (grant no. 2014JSJJ034) and Innovation Project of Central South University (grant no. xcx201990539).

\section{Availability of data and materials}

Not applicable.

\section{Authors' contributions}

LT conceived and designed the study. YM, YL, AL, HZ and LT discussed, wrote and edited the manuscript. YL edited the figures. LT revised the manuscript and acquired the financial support for the project leading to this publication. LT, YM and YL confirm the authenticity of all the raw data. All authors read and approved the final manuscript.

\section{Ethics approval and consent to participate}

Not applicable. 


\section{Patient consent for publication}

Not applicable.

\section{Competing interests}

The authors declare that they have no competing interests.

\section{References}

1. Memczak S, Jens M, Elefsinioti A, Torti F, Krueger J, Rybak A, Maier L, Mackowiak SD, Gregersen LH, Munschauer M, et al: Circular RNAs are a large class of animal RNAs with regulatory potency. Nature 495: 333-338, 2013.

2. Chen M, Xu Y, Hong N, Yang Y, Lei W, Du L, Zhao J, Lei X, Xiong L, Cai L, et al: Epidemiology of fungal infections in China. Front Med 12: 58-75, 2018.

3. Tan KE and Lim YY: Viruses join the circular RNA world FEBS J: Nov 25, 2020 (Epub ahead of print).

4. Xie H, Sun H, Mu R, Li S, Li Y, Yang C, Xu M, Duan X and Chen L: The role of circular RNAs in viral infection and related diseases. Virus Res 291: 198205, 2021.

5. Garassino MC, Whisenant JG, Huang LC, Trama A, Torri V, Agustoni F, Baena J, Banna G, Berardi R, Bettini AC, et al: COVID-19 in patients with thoracic malignancies (TERAVOLT): First results of an international, registry-based, cohort study. Lancet Oncol 21: 914-922, 2020.

6. Sun X, Wang L, Ding J, Wang Y, Wang J, Zhang X, Che Y, Liu Z, Zhang X, Ye J, et al: Integrative analysis of Arabidopsis thaliana transcriptomics reveals intuitive splicing mechanism for circular RNA. FEBS Lett 590: 3510-3516, 2016.

7. Jeck WR, Sorrentino JA, Wang K, Slevin MK, Burd CE, Liu J, Marzluff WF and Sharpless NE: Circular RNAs are abundant, conserved, and associated with ALU repeats. RNA 19: 141-157, 2013.

8. Zhang Y, Xue W, Li X, Zhang J, Chen S, Zhang JL, Yang L and Chen LL: The biogenesis of nascent circular RNAs. Cell Rep 15: 611-624, 2016.

9. Liang D, Tatomer DC, Luo Z, Wu H, Yang L, Chen LL, Cherry S and Wilusz JE: The output of protein-coding genes shifts to circular RNAs when the Pre-mRNA processing machinery is limiting. Mol Cell 68: 940-954.e3, 2017.

10. Li X, Liu CX, Xue W, Zhang Y, Jiang S, Yin QF, Wei J, Yao RW, Yang L and Chen LL: Coordinated circRNA biogenesis and function with NF90/NF110 in viral infection. Mol Cell 67: 214-227.e7, 2017.

11. Li X, Yang L and Chen LL: The biogenesis, functions, and challenges of circular RNAs. Mol Cell 71: 428-442, 2018.

12. Zou Y, Zheng S, Deng X, Yang A, Xie X, Tang H and Xie X: The role of circular RNA CDR1as/ciRS-7 in regulating tumor microenvironment: A pan-cancer analysis. Biomolecules 9: 429, 2019.

13. Akhter R: Circular RNA and Alzheimer's disease. Adv Exp Med Biol 1087: 239-243, 2018.

14. Xiao R, Chen JY, Liang Z, Luo D, Chen G, Lu ZJ, Chen Y, Zhou B, Li H, Du X, et al: Pervasive chromatin-RNA binding protein interactions enable RNA-Based regulation of transcription. Cell 178: 107-121.e18, 2019.

15. Ule J and Blencowe BJ: Alternative splicing regulatory networks: Functions, mechanisms, and evolution. Mol Cell 76: 329-345, 2019.

16. Williams T, Ngo LH and Wickramasinghe VO: Nuclear export of RNA: Different sizes, shapes and functions. Semin Cell Dev Biol 75: 70-77, 2018.

17. Martinez-Salas E, Lozano G, Fernandez-Chamorro J, Francisco-Velilla R, Galan A and Diaz R: RNA-binding proteins impacting on internal initiation of translation. Int J Mol Sci 14: 21705-21726, 2013.

18. Liu KS, Pan F, Mao XD, Liu C and Chen YJ: Biological functions of circular RNAs and their roles in occurrence of reproduction and gynecological diseases. Am J Transl Res 11: 1-15, 2019.

19. Legnini I, Di Timoteo G, Rossi F, Morlando M, Briganti F, Sthandier O, Fatica A, Santini T, Andronache A, Wade M, et al: Circ-ZNF609 is a circular RNA that can be translated and functions in myogenesis. Mol Cell 66: 22-37.e9, 2017.
20. Pamudurti NR, Bartok O, Jens M, Ashwal-Fluss R, Stottmeister C, Ruhe L, Hanan M, Wyler E, Perez-Hernandez D, Ramberger E, et al: Translation of CircRNAs. Mol Cell 66: 9-21.e7, 2017.

21. Ashwal-Fluss R, Meyer M, Pamudurti NR, Ivanov A, Bartok O, Hanan M, Evantal N, Memczak S, Rajewsky N and Kadener S: CircRNA biogenesis competes with pre-mRNA splicing. Mol Cell 56: 55-66, 2014.

22. Zhang Y, Yang L and Chen LL: Life without A tail: New formats of long noncoding RNAs. Int J Biochem Cell Biol 54: 338-349, 2014.

23. Li Z, Huang C, Bao C, Chen L, Lin M, Wang X, Zhong G, Yu B, $\mathrm{Hu}$ W, Dai L, et al: Exon-intron circular RNAs regulate transcription in the nucleus. Nat Struct Mol Biol 22: 256-264, 2015.

24. Awan FM, Yang BB, Naz A, Hanif A, Ikram A, Obaid A, Malik A, Janjua HA, Ali A and Sharif S: The emerging role and significance of circular RNAs in viral infections and antiviral immune responses: Possible implication as theranostic agents. RNA Biol 18: 1-15, 2021.

25. Zhao T, Zheng Y, Hao D, Jin X, Luo Q, Guo Y, Li D, Xi W, $\mathrm{Xu}$ Y, Chen Y, et al: Blood circRNAs as biomarkers for the diagnosis of community-acquired pneumonia. J Cell Biochem 120: 16483-16494, 2019.

26. Trepo C, Chan HL and Lok A: Hepatitis B virus infection. Lancet 384: 2053-2063, 2014.

27. Schweitzer A, Horn J, Mikolajczyk RT, Krause G and Ott JJ: Estimations of worldwide prevalence of chronic hepatitis B virus infection: A systematic review of data published between 1965 and 2013. Lancet 386: 1546-1555, 2015.

28. Zhou TC, Li X, Chen LJ, Fan JH, Lai X, Tang Y, Zhang L and Wei J: Differential expression profile of hepatic circular RNAs in chronic hepatitis B. J Viral Hepat 25: 1341-1351, 2018

29. Wang L, Zhao J, Ren J, Hall KH, Moorman JP, Yao ZQ and Ning S: Protein phosphatase 1 abrogates IRF7-mediated type I IFN response in antiviral immunity. Eur J Immunol 46: 2409-2419, 2016

30. Belloni L, Allweiss L, Guerrieri F, Pediconi N, Volz T, Pollicino T, Petersen J, Raimondo G, Dandri M and Levrero M: IFN- $\alpha$ inhibits HBV transcription and replication in cell culture and in humanized mice by targeting the epigenetic regulation of the nuclear cccDNA minichromosome. J Clin Invest 122: 529-537, 2012.

31. Fan H, Lv P, Lv J, Zhao X, Liu M, Zhang G and Tang H: MiR-370 suppresses HBV gene expression and replication by targeting nuclear factor IA. J Med Virol 89: 834-844, 2017.

32. Chen $\mathrm{C}$, Wu M, Zhang W, Lu W, Zhang M, Zhang Z, Zhang X and Yuan Z: MicroRNA-939 restricts hepatitis B virus by targeting Jmjd3-mediated and C/EBP $\alpha$-coordinated chromatin remodeling. Sci Rep 6: 35974, 2016

33. Shi J, Zhao J, Zhang X, Cheng Y, Hu J, Li Y, Zhao X, Shang Q, Sun Y, Tu B, et al: Activated hepatic stellate cells impair NK cell anti-fibrosis capacity through a TGF- $\beta$-dependent emperipolesis in HBV cirrhotic patients. Sci Rep 7: 44544, 2017.

34. Song G, Jia H, Xu H, Liu W, Zhu H, Li S, Shi J, Li Z, He J and Chen Z: Studying the association of microRNA-210 level with chronic hepatitis B progression. J Viral Hepat 21: 272-280, 2014.

35. Zhang GL,Li YX,Zheng SQ,Liu M,Li X and Tang H: Suppression of hepatitis B virus replication by microRNA-199a-3p and microRNA-210. Antiviral Res 88: 169-175, 2010.

36. Li J, Zhang X, Chen L, Zhang Z, Zhang J, Wang W, Wu M, Shi B, Zhang X, Kozlowski M, et al: Circulating miR-210 and miR-22 combined with ALT predict the virological response to interferon-alpha therapy of CHB patients. Sci Rep 7: 15658, 2017.

37. Zhang L and Wang Z: Circular RNA hsa_circ_0004812 impairs IFN-induced immune response by sponging miR-1287-5p to regulate FSTL1 in chronic hepatitis B. Virol J 17: 40, 2020.

38. Yang MH, Chen M, Mo HH, Tsai WC, Chang YC, Chang CC, Chen $\mathrm{KC}$, Wu HY, Yuan $\mathrm{CH}$, Lee $\mathrm{CH}$, et al: Utilizing experimental mouse model to identify effectors of hepatocellular carcinoma induced by HBx antigen. Cancers (Basel) 12: 409, 2020.

39. Li Y, Gao X, Wang Z, Liu W, Xu F, Hu Y, Li Y and Shi L: Circular RNA 4099 aggravates hydrogen peroxide-induced injury by down-regulating microRNA-706 in L02 cells. Life Sci 241: 116826, 2020.

40. Yin R, Guo D, Zhang S and Zhang X: miR-706 inhibits the oxidative stress-induced activation of PKC $\alpha /$ TAOK1 in liver fibrogenesis. Sci Rep 6: 37509, 2016.

41. Yu J, Ding WB, Wang MC, Guo XG, Xu J, Xu QG, Yang Y, Sun SH, Liu JF, Qin LX, et al: Plasma circular RNA panel to diagnose hepatitis B virus-related hepatocellular carcinoma: A large-scale, multicenter study. Int J Cancer 146: 1754-1763, 2020. 
42. Kohno T, Tsuge M, Murakami E, Hiraga N, Abe H, Miki D, Imamura $\mathrm{M}$, Ochi $\mathrm{H}$, Hayes $\mathrm{CN}$ and Chayama $\mathrm{K}$ : Human microRNA hsa-miR-1231 suppresses hepatitis B virus replication by targeting core mRNA. J Viral Hepat 21: e89-e97, 2014.

43. Zhu K, Zhan H, Peng Y, Yang L, Gao Q, Jia H, Dai Z, Tang Z, Fan J and Zhou J: Plasma hsa_circ_0027089 is a diagnostic biomarker for hepatitis B virus-related hepatocellular carcinoma. Carcinogenesis 41: 296-302, 2020.

44. Hu W, Wang X, Ding X, Li Y, Zhang X, Xie P, Yang J and Wang S: MicroRNA-141 represses HBV replication by targeting PPARA. PLoS One 7: e34165, 2012.

45. Huang XY, Huang ZL, Xu YH, Zheng Q, Chen Z, Song W, Zhou J, Tang ZY and Huang XY: Comprehensive circular RNA profiling reveals the regulatory role of the circRNA-100338/miR-141-3p pathway in hepatitis B-related hepatocellular carcinoma. Sci Rep 7: 5428, 2017.

46. Sekiba K, Otsuka M, Ohno M, Kishikawa T, Yamagami M, Suzuki T, Ishibashi R, Seimiya T, Tanaka E and Koike K: DHX9 regulates production of hepatitis $B$ virus-derived circular RNA and viral protein levels. Oncotarget 9: 20953-20964, 2018.

47. Ungerleider NA, Tibbetts SA, Renne R and Flemington EK: Gammaherpesvirus RNAs come full circle. mBio 10: e00071-19, 2019.

48. Toptan T, Abere B, Nalesnik MA, Swerdlow SH, Ranganathan S, Lee N, Shair KH, Moore PS and Chang Y: Circular DNA tumor viruses make circular RNAs. Proc Natl Acad Sci USA 115: E8737-E8745, 2018

49. Ungerleider NA, Jain V, Wang Y, Maness NJ, Blair RV, Alvarez X, Midkiff C, Kolson D, Bai S, Roberts C, et al: Comparative analysis of gammaherpesvirus circular RNA repertoires: Conserved and unique viral circular RNAs. J Virol 93: e01952-18, 2019.

50. Tagawa T, Gao S, Koparde VN, Gonzalez M, Spouge JL, Serquiña AP, Lurain K, Ramaswami R, Uldrick TS, Yarchoan R and Ziegelbauer JM: Discovery of Kaposi's sarcoma herpesvirus-encoded circular RNAs and a human antiviral circular RNA. Proc Natl Acad Sci USA 115: 12805-12810, 2018.

51. Abere B, Li J, Zhou H, Toptan T, Moore PS and Chang Y: Kaposi's sarcoma-associated herpesvirus-encoded circR NAs are expressed in infected tumor tissues and are incorporated into virions. mBio 11: e03027-19, 2020.

52. Preusser C, Hung LH, Schneider T, Schreiner S, Hardt M, Moebus A, Santoso S and Bindereif A: Selective release of circRNAs in platelet-derived extracellular vesicles. J Extracell Vesicles 7: 1424473, 2018.

53. Shi J, Hu N, Mo L, Zeng Z, Sun J and Hu Y: Deep RNA sequencing reveals a repertoire of human fibroblast circular RNAs associated with cellular responses to herpes simplex virus 1 infection. Cell Physiol Biochem 47: 2031-2045, 2018.

54. Huang JT, Chen JN, Gong LP, Bi YH, Liang J, Zhou L, He D and Shao CK: Identification of virus-encoded circular RNA. Virology 529: 144-151, 2019.

55. Sun G, Shi L, Yan S, Wan Z, Jiang N, Fu L, Li M and Guo J: MiR-15b targets cyclin D1 to regulate proliferation and apoptosis in glioma cells. Biomed Res Int 2014: 687826, 2014

56. Lovat F, Fassan M, Gasparini P, Rizzotto L, Cascione L, Pizzi M, Vicentini C, Balatti V, Palmieri D, Costinean S and Croce CM: miR-15b/16-2 deletion promotes B-cell malignancies. Proc Natl Acad Sci USA 112: 11636-11641, 2015.

57. Qiao Y, Zhao X, Liu J and Yang W: Epstein-Barr virus circRNAome as host miRNA sponge regulates virus infection, cell cycle, and oncogenesis. Bioengineered 10: 593-603, 2019.

58. Vincent HA, Ziehr B, Lenarcic EM and Moorman NJ: Human cytomegalovirus pTRS1 stimulates cap-independent translation. Virology 537: 246-253, 2019.

59. Lou YY, Wang QD, Lu YT, Tu MY, Xu X, Xia Y, Peng Y,Lai MM and Zheng XQ: Differential circRNA expression profiles in latent human cytomegalovirus infection and validation using clinical samples. Physiol Genomics 51: 51-58, 2019.

60. Zhang Y, Zhang H, An M, Zhao B, Ding H, Zhang Z, He Y, Shang $\mathrm{H}$ and Han $\mathrm{X}$ : Crosstalk in competing endogenous RNA networks reveals new circular RNAs involved in the pathogenesis of early HIV infection. J Transl Med 16: 332, 2018.

61. Shi B, Sharifi HJ, DiGrigoli S, Kinnetz M, Mellon K, Hu W and de Noronha CMC: Inhibition of HIV early replication by the p53 and its downstream gene p21. Virol J 15: 53, 2018.

62. Khan SZ and Mitra D: Cyclin K inhibits HIV-1 gene expression and replication by interfering with cyclin-dependent kinase 9 (CDK9)-cyclin T1 interaction in Nef-dependent manner. J Biol Chem 286: 22943-22954, 2011.
63. Manganaro L, Hong P, Hernandez MM, Argyle D, Mulder LCF, Potla U, Diaz-Griffero F, Lee B, Fernandez-Sesma A and Simon V: IL-15 regulates susceptibility of $\mathrm{CD}^{+} \mathrm{T}$ cells to HIV infection. Proc Natl Acad Sci USA 115: E9659-E9667, 2018.

64. Zhao J, Lee EE, Kim J, Yang R, Chamseddin B, Ni C, Gusho E, Xie Y, Chiang CM, Buszczak M, et al: Transforming activity of an oncoprotein-encoding circular RNA from human papillomavirus. Nat Commun 10: 2300, 2019.

65. Chan JF, Lau SK, To KK, Cheng VC, Woo PC and Yuen KY: Middle East respiratory syndrome coronavirus: Another zoonotic betacoronavirus causing SARS-like disease. Clin Microbiol Rev 28: 465-522, 2015.

66. Zumla A, Chan JF, Azhar EI, Hui DS and Yuen KY: Coronaviruses-drug discovery and therapeutic options. Nat Rev Drug Discov 15: 327-347, 2016.

67. Zhang X, Chu H, Wen L, Shuai H, Yang D, Wang Y, Hou Y, Zhu Z, Yuan S, Yin F, et al: Competing endogenous RNA network profiling reveals novel host dependency factors required for MERS-CoV propagation. Emerg Microbes Infect 9: 733-746, 2020.

68. Morrison DK: MAP kinase pathways. Cold Spring Harb Perspect Biol 4: a011254, 2012

69. Kindrachuk J, Ork B, Hart BJ, Mazur S, Holbrook MR Frieman MB, Traynor D, Johnson RF, Dyall J, Kuhn JH, et al: Antiviral potential of ERK/MAPK and PI3K/AKT/mTOR signaling modulation for Middle East respiratory syndrome coronavirus infection as identified by temporal kinome analysis. Antimicrob Agents Chemother 59: 1088-1099, 2015.

70. Echterdiek F, Kitterer D, Alscher MD, Schwenger V, Ruckenbrod B, Bald M and Latus J: Clinical course of hantavirus-induced nephropathia epidemica in children compared to adults in Germany-analysis of 317 patients. Pediatr Nephrol 34: 1247-1252, 2019.

71. Lu S, Zhu N, Guo W, Wang X, Li K, Yan J, Jiang C, Han S, Xiang H, Wu X, et al: RNA-Seq revealed a circular RNA-microRNA-mRNA regulatory network in hantaan virus infection. Front Cell Infect Microbiol 10: 97, 2020.

72. Power D, Santoso N, Dieringer M, Yu J, Huang H, Simpson S, Seth I, Miao H and Zhu J: IFI44 suppresses HIV-1 LTR promoter activity and facilitates its latency. Virology 481: 142-150, 2015.

73. DeDiego ML, Nogales A, Martinez-Sobrido L and Topham DJ: Interferon-induced protein 44 interacts with cellular FK506-binding protein 5 , negatively regulates host antiviral responses, and supports virus replication. mBio 10: e1839-19, 2019.

74. Melchjorsen J, Kristiansen H, Christiansen R, Rintahaka J, Matikainen S, Paludan SR and Hartmann R: Differential regulation of the OASL and OAS1 genes in response to viral infections. J Interferon Cytokine Res 29: 199-207, 2009.

75. Leung DW: Mechanisms of non-segmented negative sense RNA viral antagonism of host RIG-I-Like receptors. J Mol Biol 431: 4281-4289, 2019.

76. Lupfer C, Malik A and Kanneganti TD: Inflammasome control of viral infection. Curr Opin Virol 12: 38-46, 2015.

77. Arora S, Singh P, Dohare R, Jha R and Ali Syed M: Unravelling host-pathogen interactions: ceRNA network in SARS-CoV-2 infection (COVID-19). Gene 762: 145057, 2020.

78. Farberov L, Herzig E, Modai S, Isakov O, Hizi A and Shomron N: MicroRNA-mediated regulation of $\mathrm{p} 21$ and TASK1 cellular restriction factors enhances HIV-1 infection. J Cell Sci 128: 1607-1616, 2015.

79. Yu T, Ding Y, Zhang Y, Liu Y, Li Y, Lei J, Zhou J, Song S and $\mathrm{Hu}$ B: Circular RNA GATAD2A promotes H1N1 replication through inhibiting autophagy. Vet Microbiol 231: 238-245, 2019.

80. Wang ZY, Guo ZD, Li JM, Zhao ZZ, Fu YY, Zhang CM, Zhang Y, Liu LN, Qian J and Liu LN: Genome-wide search for competing endogenous RNAs responsible for the effects induced by ebola virus replication and transcription using a trVLP system. Front Cell Infect Microbiol 7: 479, 2017.

81. Yao F, Kausalya JP, Sia YY, Teo AS, Lee WH, Ong AG, Zhang Z, Tan JH, Li G, Bertrand D, et al: Recurrent fusion genes in gastric cancer: CLDN18-ARHGAP26 induces loss of epithelial integrity. Cell Rep 12: 272-285, 2015.

82. Barba-Spaeth G, Dejnirattisai W, Rouvinski A, Vaney MC, Medits I, Sharma A, Simon-Lorière E, Sakuntabhai A, Cao-Lormeau VM, Haouz A, et al: Structural basis of potent Zika-dengue virus antibody cross-neutralization. Nature 536: 48-53, 2016.

83. Swanstrom JA, Plante JA, Plante KS, Young EF, McGowan E, Gallichotte EN, Widman DG, Heise MT, de Silva AM and Baric RS: Dengue virus envelope dimer epitope monoclonal antibodies isolated from dengue patients are protective against Zika virus. mBio 7: e01123-16, 2016. 
84. He J,Ming Y,MinLi Y,Han Z, Jiang J,Zhou J,Dai B,Lv Y,He ML, Fang $M$ and Li Y: hsa_circ_0006459 and hsa_circ_0015962 affect prognosis of dengue fever. Sci Rep 9: 19425, 2019.

85. Zhao K, Zhao Q, Guo Z, Chen Z, Hu Y, Su J, Chen L, He Z, Cai X, Chen M, et al: Hsa_Circ_0001275: A potential novel diagnostic biomarker for postmenopausal osteoporosis. Cell Physiol Biochem 46: 2508-2516, 2018.

86. Liu Q, Shuai M and Xia Y: Knockdown of EBV-encoded circRNA circRPMS1 suppresses nasopharyngeal carcinoma cell proliferation and metastasis through sponging multiple miRNAs. Cancer Manag Res 11: 8023-8031, 2019.

87. Shuai M and Huang L: High expression of hsa_circRNA_001387 in nasopharyngeal carcinoma and the effect on efficacy of radiotherapy. Onco Targets Ther 13: 3965-3973, 2020.

88. Zhou Y, Shen L, Wang YZ and Zhou CC: The potential of ciRS-7 for predicting onset and prognosis of cervical cancer. Neoplasma 67: 312-322, 2020.

89. Wang M, Gu B, Yao G, Li P and Wang K: Circular RNA expression profiles and the pro-tumorigenic function of CircRNA_10156 in Hepatitis B virus-related liver cancer. Int J Med Sci 17: 1351-1365, 2020.

90. Chen J, Chan AW, To KF, Chen W, Zhang Z, Ren J, Song C, Cheung YS, Lai PB, Cheng SH, et al: SIRT2 overexpression in hepatocellular carcinoma mediates epithelial to mesenchymal transition by protein kinase B/glycogen synthase kinase- $3 \beta /$ $\beta$-catenin signaling. Hepatology 57: 2287-2298, 2013.

91. Guo H, Zhou T, Jiang D, Cuconati A, Xiao GH, Block TM and Guo JT: Regulation of hepatitis B virus replication by the phosphatidylinositol 3-kinase-akt signal transduction pathway. J Virol 81: 10072-10080, 2007.

92. Jiang W, Wang L, Zhang Y and Li H: Circ-ATP5H induces hepatitis $\mathrm{B}$ virus replication and expression by regulating miR-138-5p/TNFAIP3 axis. Cancer Manag Res 12: 11031-11040, 2020.
93. Cheung CC, Chung GT, Lun SW, To KF, Choy KW, Lau KM, Siu SP, Guan XY, Ngan RK, Yip TT, et al: miR-31 is consistently inactivated in EBV-associated nasopharyngeal carcinoma and contributes to its tumorigenesis. Mol Cancer 13: 184, 2014.

94. Liu N, Jiang N, Guo R, Jiang W, He QM, Xu YF, Li YQ, Tang LL, Mao YP, Sun Y and Ma J: MiR-451 inhibits cell growth and invasion by targeting MIF and is associated with survival in nasopharyngeal carcinoma. Mol Cancer 12: 123, 2013.

95. Ungerleider N, Concha M, Lin Z, Roberts C, Wang X, Cao S, Baddoo M, Moss WN, Yu Y, Seddon M, et al: The Epstein Barr virus circRNAome. PLoS Pathog 14: e1007206, 2018.

96. Gong LP, Chen JN, Dong M, XiaoZD, Feng ZY, Pan YH,Zhang Y, Du Y, Zhang JY, Bi YH, et al: Epstein-Barr virus-derived circular RNA LMP2A induces stemness in EBV-associated gastric cancer. EMBO Rep 21: e49689, 2020.

97. Li Y, Masaki T, Yamane D, McGivern DR and Lemon SM: Competing and noncompeting activities of miR-122 and the 5 exonuclease Xrn1 in regulation of hepatitis $\mathrm{C}$ virus replication. Proc Natl Acad Sci USA 110: 1881-1886, 2013.

98. Jost I, Shalamova LA, Gerresheim GK, Niepmann M, Bindereif A and Rossbach O: Functional sequestration of microRNA-122 from hepatitis $\mathrm{C}$ virus by circular RNA sponges. RNA Biol 15: 1032-1039, 2018

This work is licensed under a Creative Commons Attribution-NonCommercial-NoDerivatives 4.0 International (CC BY-NC-ND 4.0) License. 